\title{
Fejer-Riesz Theorem and Its Generalization
}

\author{
Abdulmtalb Hussen*, Abdelbaset Zeyani ${ }^{* *}$ \\ *Maryville University, \\ ** University of Missouri-St. Louis,
}

\begin{abstract}
The classical Fejer-Riesz Theorem has many applications in various mathematical fields. This survey paper presents this theorem in several versions: 1) with operatorvalued functions as coefficients, 2) in $2 \times 2$ matrix form, and 3 ) in the multivariate case. The main ideas of the proofs are sketched.
\end{abstract}

Index Terms- Fejer-Riesz Theorem, Laurent Polynomial, Fractionation, Operator theory, Multivariate Polynomials.

\section{INTRODUCTION}

A Laurent polynomial is an expression of the form

$$
P(z)=\sum_{k=-M_{1}}^{M_{2}} c_{k} z^{k},
$$

for some integers $M_{1}$ and $M_{2}$.

$$
\begin{aligned}
& \text { When } z=e^{-i \theta} \text {, } \\
& \qquad P\left(e^{-i \theta}\right)=\sum_{k=-M_{1}}^{M_{2}} c_{k} e^{-i \theta k},
\end{aligned}
$$

is a trigonometric polynomial for some integers $M_{1}$ and $M_{2}$. If $P\left(e^{-i \theta}\right)$ values are real for all $\theta \in \mathbb{R}$, then the coefficients $c_{k}$ must be real and $c_{k}=\bar{c}_{-k}$ for all $k$.

At the beginning of the nineteen century Fejer in [9] and Riesz in [13] have shown that the nonnegative trigonometric polynomial in (2) with real coefficients can be written as a square polynomial i.e

$$
P\left(e^{-i \theta}\right)=\left|Q\left(e^{-i \theta}\right)\right|^{2}
$$

for some polynomial

$$
Q(z)=\sum_{k=0}^{M_{2}} c_{k} z^{k}
$$

This result is called Fejer-Riesz Theorem. There exist many different proofs of this Theorem [4, 6, 7, 11, 14-16].

A more general version of Fejer-Riesz Theorem takes the form of operator-valued functions, which means the coefficients in (1) are bounded operators in some Hilbert space.
Also, this result has been generalized to the matrix case.

There also exists a generalization of the Fejer-Riesz Theorem to several variables. A multivariate Laurent polynomial that is strictly positive on the unit torus can be factored into a sum of hermitian squares values of other polynomials $[6,10,11]$.

This survey paper presents the classical Fejer-Riesz Theorem with real and complex coefficients and outlines its proof. Also, we give different versions of Fejer-Riesz Theorem in the operator form, matrix case and multivariate case.

\section{FEJER-RIESZ THEOREM IN DIFFERENT VERSIONS}

In this section, we present the classical Fejer-Riesz Theorem and some of its generalization.

\section{A. Classical Fejer-Riesz Theorem}

Theorem 1. Let

$$
A(\xi)=\sum_{k=0}^{M} a_{k} \cos (k \xi),
$$

be a trigonometric polynomial with real coefficients, which is nonnegative for every real number $\xi$. Then there exists a trigonometric polynomial $B$ of order $M$. i.e

$$
B\left(e^{i \xi}\right)=\sum_{k=0}^{M} b_{k} e^{i k \xi}, \quad b_{k} \in \mathbb{R}
$$

such that

$$
A(\xi)=|B(\xi)|^{2}
$$

In addition, for some $B(z)=\sum_{k=0}^{M} a_{k} z^{k}$, the polynomial $B(z)$ can be chosen to have no roots in $D=\{z \in \mathbb{C}:|z|<1\}$ [5].

The proof of this theorem is based on the factorization of $A(\xi)$. By the fundamental theorem of algebra, (5) can be factored as

$$
p_{A}(c)=\alpha \prod_{j=1}^{M}\left(c-c_{j}\right)
$$


where $c=\frac{z+z^{-1}}{2}, z=e^{-i \xi}, \alpha=\frac{1}{2} a_{M}$, and the roots $c_{j}$ of $p_{A}$ appears either in conjugate pairs $c_{j}, \bar{c}_{j}$, or in real singletons. If $a_{M} \neq 0$, then $A(\xi)=e^{M i \xi} P_{A}\left(e^{-i \xi}\right)$, where $P_{A}$ is a polynomial of degree $2 M$ with $P_{A}(0) \neq 0 . P(z)$ roots occur in reciprocal pairs $z_{j}$ and $\frac{1}{\bar{z}_{j}}$ with $\left|z_{j}\right| \geq 1$. In particular, the roots in the unit circle $\mathbb{T}$ must have even multiplicity; it follows that

$$
\begin{aligned}
P_{A}(z) & =\frac{1}{2} a_{M}\left[\prod_{k=1}^{K}\left(z-r_{k}\right)\left(z-\overline{r_{k}}\right)\right] \times \\
& {\left[\prod_{j=1}^{J}\left(z-z_{j}\right)\left(z-\overline{z_{j}}\right)\left(z-z_{j}^{-1}\right)\left(z-\bar{z}_{j}^{-1}\right)\right], }
\end{aligned}
$$

where $r_{k} \in \mathbb{R} /\{0\}, k=1,2,3, \ldots, K$ and $z_{j} \in \mathbb{C} / \mathbb{R}, j=$ $1,2,3, \ldots J$ and $K+2 J=M$. It follows that

$$
\begin{aligned}
A(\xi) & =C\left|\prod_{k=1}^{K}\left(e^{-i \xi}-r_{k}\right) \prod_{j=1}^{J}\left(e^{-i \xi}-z_{j}\right)\left(e^{-i \xi}-\overline{z_{j}}\right)\right|^{2} \\
& =|B(\xi)|^{2}
\end{aligned}
$$

where $C$ is a positive constant, and therefore the desired result is obtained as

$$
B(\xi)=\sqrt{C} \prod_{k=1}^{K}\left(e^{-i \xi}-r_{k}\right) \prod_{j=1}^{J}\left(e^{-i \xi}-z_{j}\right)\left(e^{-i \xi}-\overline{z_{j}}\right)
$$

with $K+2 J=M$, see [2, 3, 12].

Remark The polynomial $B$ in (6) is not unique because we can choose any root from every pair reciprocals of roots of $P_{A}(z)$ to get $B(\xi)$. Let's look at an example

Suppose

$$
A(\xi)=2-\cos (\xi)
$$

be a trigonometric polynomial with real coefficients $a_{0}=2$ and $a_{1}=-1$ and with $M=1$. Then clearly $A$ is a real on $\mathbb{T}$, and also one can check that $A$ is nonnegative on $\mathbb{T}$. Then we can get the polynomial $P_{A}(z)$ as

$$
\begin{aligned}
P_{A}(z) & =\frac{1}{2}\left(-1+4 z-z^{2}\right) \\
& =-\frac{1}{2}(z-(2-\sqrt{3}))(z-(2+\sqrt{3})) .
\end{aligned}
$$

If we choose the zero outside the unit circle, then by using (11) we get,

$$
\begin{aligned}
B(z) & =\left(\frac{1}{2}\left|a_{1}\right|\left|(2+\sqrt{3})^{-1}\right|^{1 / 2}\right) \times(z-(2+\sqrt{3})) \\
& =\frac{1}{\sqrt{2}}\left(\frac{1}{\sqrt{2+\sqrt{3}}}\right)(z-(2+\sqrt{3})) \\
& =\frac{1}{2}[(\sqrt{3}-1) z-(\sqrt{3}+1)] .
\end{aligned}
$$

Thus

$$
|B(\xi)|^{2}=A(\xi)
$$

Now, we present the complex version of the Fejer-Riesz Theorem.
Theorem 2. Let

$$
A(z)=\sum_{k=-N}^{N} a_{k} z^{k}
$$

such that, $a_{0} \in \mathbb{R}, a_{k} \in \mathbb{C} \quad k=1,2, \ldots, N$. If $A(z) \geq 0$ on $|z|=1$, then there exists a polynomial

$$
B(z)=\sum_{k=0}^{N} b_{k} z^{k}
$$

where $b_{k} \in \mathbb{C}$, for $k=0,1, \ldots, N$, such that

$$
A(z)=\left(\sum_{k=0}^{N} b_{k} z^{k}\right)\left(\sum_{k=0}^{N} \overline{b_{k}} z^{-k}\right)
$$

To prove Theorem (2), we use induction . Let us start with the simplest case i.e. , $N=1$. We need to consider two cases: the roots are on the unit circle or not on the unit circle.

1. If the roots $z_{1}, \bar{z}_{1}^{-1}$ are not on the unit circle, we get

$$
\begin{aligned}
A(z) & =a_{0}+a_{1} z+\overline{a_{1}} z^{-1} \\
& =z^{-1}\left[\overline{a_{1}}+a_{0} z+a_{1} z^{2}\right] \\
& =-\frac{a_{1}}{\overline{z_{1}}}\left(1-z_{1} z^{-1}\right)\left(1-\overline{z_{1}} z\right)
\end{aligned}
$$

for every $z \in \mathbb{C}$ such that $|z|=1$, we can see

$$
-\frac{a_{1}}{\overline{z_{1}}}=\frac{A(z)}{\left(1-z_{1} z^{-1}\right)\left(1-\overline{z_{1}} z\right)}=\frac{A(z)}{\left|1-z_{1} z\right|^{2}} \geq 0 .
$$

Therefore we let

$$
B(z)=\sqrt{-\frac{a_{1}}{\overline{z_{1}}}}\left(1-\overline{z_{1}} z\right) .
$$

One can check that

$$
A(z)=B(z) \bar{B}\left(z^{-1}\right)
$$

2. If the roots $z_{0},{\overline{z_{0}}}^{-1}$ are on the unit circle, in other words, $A\left(z_{0}\right)=0$ and $\left|z_{0}\right|=1$ then we can get,

$$
\begin{aligned}
A(z) & =a_{0}+a_{1} z+\overline{a_{1}} z^{-1} \\
& =a_{1} z^{-1}\left(z-z_{0}\right)\left(z-\bar{z}_{0}-1\right) \\
& =-\frac{a_{1}}{\overline{z_{0}}}\left(z-z_{0}\right)\left(z^{-1}-\overline{z_{0}}\right) .
\end{aligned}
$$

Observe that $-\frac{a_{1}}{\overline{z_{0}}} \geq 0$ due to $A(z) \geq 0$ on $|z|=1$.

Therefore, we can take

$$
B(z)=\sqrt{-\frac{a_{1}}{\overline{z_{1}}}}\left(z-z_{0}\right),
$$

which satisfies (17). 
Now, let us assume that $(15)$ is true when $N=M$ i.e

$$
\begin{aligned}
& \tilde{A}(z)=\sum_{k=-M}^{M} \tilde{a}_{k} z^{k}, \\
& \text { for } \tilde{a}_{0} \in \mathbb{R}, \quad \tilde{a}_{k} \in \mathbb{C} \quad k=1,2, \ldots, M .
\end{aligned}
$$

As before, we consider two cases:

1. The roots are not on the unit circle, $|z|=1$.

$$
\begin{aligned}
\tilde{\tilde{A}}(z) & =\sum_{k=-(M+1)}^{M+1} \tilde{\tilde{a}}_{k} z^{k} \\
& =C\left(1-z_{1} z^{-1}\right)\left(1-\bar{z}_{1}\right) \tilde{A}(z),
\end{aligned}
$$

where $C$ is a positive constant.

We can see

$$
\tilde{A}(z)=\frac{\tilde{\tilde{A}}(z)}{C\left(1-z_{1} z^{-1}\right)\left(1-\bar{z}_{1} z\right)} \geq 0,
$$

so,

$$
\tilde{\tilde{A}}(z)=C\left(1-z_{1} z^{-1}\right)\left(1-\bar{z}_{1} z\right) \tilde{B}(z) \overline{\tilde{B}}(z) .
$$

Therefore we can take

$$
\tilde{\tilde{B}}(z)=\sqrt{C}\left(1-\bar{z}_{1}\right) \tilde{B}(z)
$$

where $\tilde{B}(z)$ has the form $(14)$.

2. The roots are on $|z|=1$.

$$
\begin{aligned}
\tilde{\tilde{A}}(z) & =\sum_{k=-(M+1)}^{M+1} \tilde{\tilde{a}}_{k} z^{k} \\
& =C\left(z-z_{0}\right)\left(z^{-1}-\overline{z_{0}}\right) \tilde{A}(z),
\end{aligned}
$$

we also observe that $\tilde{A}(z) \geq 0$ on the unit circle, $|z|=1$, so,

$$
\tilde{\tilde{A}}(z)=C\left(z-z_{0}\right)\left(z^{-1}-\overline{z_{0}}\right) \tilde{B}(z) \overline{\tilde{B}}(z) .
$$

Therefore we can take

$$
\tilde{\tilde{B}}(z)=\sqrt{C}\left(z-z_{0}\right) \tilde{B}(z) .
$$

\section{B. Fejer-Riesz Theorem ( Operator Version)}

In this subsection, we present another version of the FejerRiesz Theorem, which is in the operator form. The result that we will obtain for this version is similar to that of Theorem (1), just with the coefficients as the operators in a Hilbert space. Before we state the Theorem, we will give some definitions and facts used in this Theorem's proof.

Let $U$ and $V$ be two vector spaces, and an operator is a mapping from $U$ to $V$. Let $\mathfrak{H}$ be a complex Hilbert space, and let $\mathfrak{B}(\mathfrak{H})$ be the set of all bounded operators from $\mathfrak{H}$ to $\mathfrak{H}$. An operator $S$ in $\mathfrak{B}(\mathfrak{H})$ is called a shift operator if $S$ is an isometry and $S^{* n} \rightarrow 0$ strongly, that is $\left\|S^{* n} f\right\| \rightarrow 0$ for all $f \in \mathfrak{H}$. We mean by an isometry operator here is a linear operator that preservers distance, i.e for all $x_{1}, x_{2} \in \mathfrak{H}$, we have

$$
\left\|S x_{1}-S x_{2}\right\|=\left\|x_{1}-x_{2}\right\| .
$$

We say that $T \in \mathfrak{B}(\mathfrak{H})$ is Toeplitz if

$$
S^{*} T S=T,
$$

and it is analytic (with respect to $S$ ) if

$$
S T=T S,
$$

where $S$ is a fixed shift operator on the Hilbert space $\mathfrak{H}$. If $T$ is analytic, then $T$ and $T^{*}$ and their linear combinations are Toeplitz.

An analytic operator $T$ is said to be outer if its range is a subspace of $\mathfrak{H}$.

Definition II.1. (Operator Matrix) An operator $T \in \mathfrak{B}(\mathfrak{H})$ is called a matrix operator in $\mathfrak{B}(\mathfrak{K})$ if $T \sim\left[A_{j k}\right]_{j, k=0}^{\infty}$ where

$$
A_{j k}=P_{0} S^{* j} T S^{k} P_{0} \mid \mathfrak{K}, \quad j, k \geq 0
$$

where $P_{0}=I-S S^{*}$.

The following theorem and its result are given in the matrix forms of Toeplitz and analytic operators $[15,16]$.

Theorem 3. Let $T \in \mathfrak{B}(\mathfrak{H})$ be an operator, then $T$ is Toeplitz if and only if its matrix has the form

$$
\left[\begin{array}{cccc}
A_{0} & A_{-1} & A_{-2} & \ldots \\
A_{1} & A_{0} & A_{-1} & \ddots \\
A_{2} & A_{1} & A_{0} & \ddots \\
\vdots & \ddots & \ddots & \ddots
\end{array}\right],
$$

where

$$
A_{j}= \begin{cases}P_{0} S^{* j} T P_{0} \mid \mathfrak{K}, & \text { if } j \geq 0 \\ P_{0} T S^{|j|} P_{0} \mid \mathfrak{K}, & \text { if } j<0\end{cases}
$$

The matrix of the form (27) is called a Toeplitz matrix.

Corollary 3.1. Let $A \in \mathfrak{B}(\mathfrak{H})$ be an operator, then $A$ is analytic if and only if its matrix has the form

$$
\left[\begin{array}{cccc}
A_{0} & 0 & 0 & \ldots \\
A_{1} & A_{0} & 0 & \ddots \\
A_{2} & A_{1} & A_{0} & \ddots \\
\vdots & \ddots & \ddots & \ddots
\end{array}\right],
$$

where

$$
A_{j}=P_{0} S^{* j} T P_{0} \mid \mathfrak{K}, \quad j \geq 0 .
$$


Now, let us introduce a new notation $\mathfrak{C}$, which is a separable Hilbert space, and $\mathfrak{B}(\mathfrak{C})$ is the set of a bounded linear operator on $\mathfrak{C}$.

An example of a Laurent polynomial is

$$
Q(z)=\sum_{k=-m}^{m} Q_{k} z^{k},
$$

and an analytic polynomial is

$$
P(z)=\sum_{k=0}^{m} P_{k} z^{k},
$$

where $Q_{k}, P_{k} \in \mathfrak{B}(\mathfrak{C})$. Set $Q_{j}=0$ for $|j|>m$ and $P_{j}=0$ for $j>m$. Then

$$
T_{Q}=\left[\begin{array}{cccc}
Q_{0} & Q_{-1} & Q_{-2} & \ldots \\
Q_{1} & Q_{0} & Q_{-1} & \ddots \\
Q_{2} & Q_{1} & Q_{0} & \ddots \\
\vdots & \ddots & \ddots & \ddots
\end{array}\right],
$$

defines the Toeplitz operator for the Laurent polynomial $Q(z)$.

Also,

$$
T_{P}=\left[\begin{array}{cccc}
P_{0} & 0 & 0 & \ldots \\
P_{1} & P_{0} & 0 & \ddots \\
P_{2} & P_{1} & P_{0} & \ddots \\
\vdots & \ddots & \ddots & \ddots
\end{array}\right],
$$

defines the analytic operator for the analytic polynomial $P(z)$. Moreover, $T_{Q}$ and $T_{P}$ are bounded Operators on $\mathfrak{C}^{\infty}$. If the analytic Toeplitz operator $A=T_{P}$ is outer, then we say that polynomial $P(z)$ is outer [7].

Lemma 4. (Lowdenslager's Isometry) Let $T \in \mathfrak{B}(\mathfrak{H})$ be a Toeplitz operator such that $T \geq 0$, then there exists a unique square root $T^{1 / 2} \geq 0$. Since $T=S^{*} T S$ Then for any $f \in \mathfrak{H}$, $T^{1 / 2} S f$ and $T^{1 / 2} f$ have the same norms [7].

Definition II.2. Let $T \in \mathfrak{B}(\mathfrak{H})$ be any nonnegative Toeplitz operator, and let $\mathfrak{H}_{T}=C L\left(T^{1 / 2} \mathfrak{H}\right)$ be the closure of the range of $T^{1 / 2}$, and it is considered as a Hilbert space with the inner product on $\mathfrak{H}$. Then there is a unique isometry $S_{T}$ on $\mathfrak{H}_{T}$, which is called Lowdenslager's Isometry, such that

$$
S_{T} T^{1 / 2} f=T^{1 / 2} S f, \quad \text { for all } \quad f \in \mathfrak{H}
$$

Theorem 5. Let $T \in \mathfrak{B}(\mathfrak{H})$ be a nonnegative Toeplitz operator. Then $T$ is factorable, i.e $T=A^{*} A$ for some analytic operator $A \in \mathfrak{B}(\mathfrak{H})$ if and only if the Lowdenslager's isometry $S_{T}$ is a shift operator $A$ can be chosen to be outer.

Lemma 6. Let $T=T_{Q}$ be the Toeplitz operator for a Laurent polynomial $Q(z)$ that is given by (33). If $T=A^{*} A$, where $A \in \mathfrak{B}(\mathfrak{H})$ is analytic and outer, then $A=T_{P}$ for some outer analytic polynomial $P(z)$ of degree $m$.
Now, we are ready to give an operator version of the FejerRiesz Theorem and how the above facts and theorems proved it.

\section{Theorem 7. (Operator Fejer-Riesz, Theorem)}

For a Laurent polynomial $Q(z)=\sum_{k=-m}^{m} Q_{k} z^{k}$ such that coefficients in $\mathfrak{B}(\mathfrak{C})$ for some Hilbert space $\mathfrak{C}$ where $z=e^{i \theta}$. We assume that $Q(z) \geq 0$ on the unit circle $\mathbb{T}$. Then there exists a polynomial $P(z)=\sum_{k=0}^{m} P_{k} z^{k}$ with coefficients in $\mathfrak{B}(\mathfrak{C})$ such that

$$
Q(z)=P^{*}(z) P(z) .
$$

The polynomial $P(z)$ can be chosen outer.

By looking for lemma (6), and Theorem (5), we can prove this theorem. We let $T=T_{Q}$ as in (33) and by showing $S_{T}$ is a shift operator, we conclude that $T=T_{P}^{*} T_{P}$. To show that $S_{T}$ is the shift operator, it suffices to show that $\left\|S_{T}^{* n} f\right\| \rightarrow 0$ for all $f \in \mathfrak{H}$.

\section{Generalization of Fejer-Riesz Theorem}

In this subsection, we will give a generalization of the FejerRiesz Theorem. The classical Fejer-Riesz Theorem can be generalized to matrix-valued Laurent polynomials.

Definition II.3. A square matrix $\mathcal{M}=\left[a_{i, j}\right]_{i, j=1}^{N} \in \mathbb{C}^{N \times N}$ is called positive semi-definite if

$$
\begin{aligned}
& \mathbf{x}^{*} \mathcal{M} \mathbf{x}=\sum_{1 \leq i, j \leq N} \overline{\mathbf{x}}_{i} \mathbf{a}_{i j} \mathbf{x}_{j} \geq 0, \\
& \text { for all } \mathbf{x}=\left(x_{1}, \ldots, x_{N}\right)^{T} \in \mathbb{C}^{N} /\{0\},
\end{aligned}
$$

and $\mathcal{M}$ is called positive definite if $\mathbf{x}^{*} \mathcal{M} \mathbf{x}>0$ for all $\mathbf{x} \in$ $\mathbb{C}^{N} /\{0\}$.

A Laurent Matrix polynomial $\mathcal{M}(z)=\sum_{k=-N}^{N} A_{k} z^{k}$ is called positive (semi-) definite on $\mathbb{T}$ if $\mathcal{M}(z)$ is positive (semi-) definite for any $z \in \mathbb{T}$. In the following theorem we will consider the coefficients $A_{k}$ are $2 \times 2$ matrix with real entries.

\section{Theorem 8. Fejer-Riesz Theorem (Matrix case) [4]}

Let

$$
\mathcal{M}(z)=\left[\begin{array}{cc}
A(z) & B(z) \\
B(1 / z) & C(z)
\end{array}\right]
$$

be a matrix of Laurent polynomials with real coefficients which is positive semidefinite on $\mathbb{T}$ and assume that $A(z)=\sum_{k=0}^{N} a_{k}(z+1 / z)^{k}, a_{N} \neq 0$, such that $A(z)$ and $B(z)$ have no common roots in $\mathbb{C} /\{0\}$. Then there exist four Laurent polynomials $u_{1}, u_{2}, v_{1}, v_{2}$ with real coefficients, with $u_{1}$ and $u_{2}$ of degree at most $N$ such that the following two conditions are equivalents.

\section{Define a factorization of $\mathcal{M}$ as:}

$$
\begin{aligned}
\mathcal{M}(z) & =\left[\begin{array}{ll}
u_{1}(1 / z) & u_{2}(1 / z) \\
v_{1}(1 / z) & v_{2}(1 / z)
\end{array}\right]\left[\begin{array}{ll}
u_{1}(z) & v_{1}(z) \\
u_{2}(z) & v_{2}(z)
\end{array}\right] \\
& =R^{T}(1 / z) R(z)
\end{aligned}
$$


2. The quadruple $\left(u_{1}, u_{2}, v_{1}, v_{2}\right)$ is a solution of the linear homogeneous system

$$
\begin{gathered}
B(z) u_{1}(z)-d(z) u_{2}(1 / z)-A(z) v_{1}(z)=0, \\
d(1 / z) u_{1}(z)+B(1 / z) u_{2}(z)-A(z) v_{2}(z)=0,
\end{gathered}
$$

and

$$
u_{1}^{2}(1)+u_{2}^{2}(1)=A(1),
$$

where dis a Laurent Polynomial such that

$$
d(z) d(1 / z)=\operatorname{det} \mathcal{M}(z) .
$$

The proof of this Theorem is divided into three parts: properties of a linear homogeneous system, factorization of $\mathcal{M}$, and existence of solutions.

We assume first that the factorization of $\mathcal{M}$ in (38) is available with $R=\left[\begin{array}{cc}u_{1} & v_{1} \\ u_{2} & v_{2} .\end{array}\right]$, where $u_{1}$ and $u_{2}$ have degrees at most $N$. The determinant of $R$ satisfies (42) and the first component of $\mathcal{M}$ in (38) satisfies (41). By letting,

$$
\begin{aligned}
& \alpha(z)=B(z) u_{1}(z)-d(z) u_{2}(1 / z)-A(z) v_{1}(z), \\
& \beta(z)=d(z) u_{1}(1 / z)+B(z) u_{2}(z)-A(z) v_{2}(z),
\end{aligned}
$$

and by using some algebraic techniques, we get a homogeneous system of linear equations for $\alpha(z)$ and $\beta(z)$ whose determinate $d(1 / z)$ is non zero for all $z \in \mathbb{T}$, i.e

$$
\left[\begin{array}{ll}
u_{1}(1 / z) & u_{2}(1 / z) \\
v_{1}(1 / z) & v_{2}(1 / z)
\end{array}\right]\left[\begin{array}{l}
\alpha(z) \\
\beta(z)
\end{array}\right]=\left[\begin{array}{l}
0 \\
0
\end{array}\right]
$$

Thus, $\alpha=\beta=0$ is the only Laurent polynomial solution, which implies to (39) and (40).

Next, by assuming the equations from (39) - (42), considering that $\alpha=\beta=0$ and taking some necessary combinations, we can get

$$
\begin{gathered}
A(z)=u_{1}(z) u_{1}(1 / z)+u_{2}(z) u_{2}(1 / z), \\
B(z)=v_{1}(z) u_{2}(1 / z)+v_{2}(z) u_{2}(1 / z), \\
C(z)=v_{1}(z) v_{1}(1 / z)+v_{2}(z) v_{2}(1 / z), \\
B(1 / z)=u_{1}(z) v_{1}(1 / z)+u_{2}(z) v_{2}(1 / z),
\end{gathered}
$$

which result in the matrix factorization (38).

Finally, we show the existence of Laurent polynomials $\left(u_{1}, u_{2}, v_{1}, v_{2}\right)$ above. The idea is simple: A linear homogeneous system has a nontrivial solution if the number of equations is less than the number of variables.

In view of (39), we need to find $u_{1}(z)$ and $u_{2}(z)$ such that

$$
B(z) u_{1}(z)-d(z) u_{2}(1 / z)
$$

is divisible by $A(z)$, which means that all roots of $A(z)$ are also the roots of $(49)$. $z^{N} A(z)$ is an algebraic polynomial which has a degree $2 N$. If $w$ is a root of $A(z)$ of multiplicity $k$, then $(z-w)^{k}$ is a factor of the Laurent polynomial (49) if and only if

$\left.\frac{d^{v}}{d z^{v}}\left[B(z) u_{1}(z)-d(z) u_{2}(1 / z)\right]\right|_{z=w}=0, \quad$ for $\quad 0 \leq v \leq k-1$.

We consider $w$ in two cases: real and complex. From this, we conclude that for the real case, each root $w$ has multiplicity $k$, we have $k$ homogeneous equations; and for the complex case that $w$ has multiplicity $k$, we have $2 k$ homogeneous equations.

Notice that $\bar{w}$ is also a root of $A(z)$; there is a one-to-one correspondence between the number of roots and the number of equations. From all the roots of $z^{N} A(z)$ we have $2 N$ linear homogeneous equations, and $2 N+2$ unknown variables because of $u_{1}(z)$ and $u_{2}(z)$, which means that the number of unknown variables is greater than the number of equations. Therefore, the non-trivial algebraic polynomials $u_{1}$ and $u_{2}$ of degrees at most $N$ exist such that $A$ divides the Laurent polynomial in (49). In other words there exists Laurent polynomials $u_{1}, u_{2}, v_{1}$ with real coefficients and $u_{1}$ and $u_{2}$ have degrees at most $N$, such that

$$
B(z) u_{1}(z)-d(z) u_{2}(1 / z)-A(z) v_{1}(z)=0
$$

The triple $\left(u_{1}, u_{2}, v_{1}\right)$ defines a solution of the equation (39). We can also show that $\left(u_{1}, u_{2}, v_{1}\right)$ leads to a solution $\left(u_{1}, u_{2}, v_{2}\right)$ of $(40)$.

\section{Multivariate of Fejer-Riesz Theorem}

The Fejer-Riesz Theorem can be extended to the multivariate case under some different conditions for Laurent polynomials. For the two-variable case, Geronimo and Woerdeman in [10] provide a necessary and sufficient condition on the degree of the polynomial. In contrast, Dritschel in [6] provides the state of the factorization of any Laurent polynomial.

Theorem 9. (Fejer-Riesz in Two Variables): Let $Q(z)$ be a polynomial, we say that $Q(z)$ is stable if $Q(z) \neq 0$ for $z \in \bar{D}=\{z \in \mathbb{C} ;|z| \leq 1\}$. The spectral density function of $Q(z)$ defined by $f(z)=(Q(z) \overline{Q(1 / \bar{z})})^{-1}$.

\section{Suppose}

$$
Q\left(z_{1}, z_{2}\right)=\sum_{l=-m}^{m} \sum_{k=-n}^{n} q_{k l} z_{1}^{k} z_{2}^{l} \text { for }\left|z_{1}\right|=\left|z_{2}\right|=1,
$$


be a Laurent polynomial in two variables, such that $Q\left(z_{1}, z_{2}\right) \geq 0$ for all $\left|z_{1}\right|=\left|z_{2}\right|=1$.

Remark: In general we can not say that there exists a polynomial

$$
P\left(z_{1}, z_{2}\right)=\sum_{l=0}^{m} \sum_{k=0}^{n} p_{k l} z_{1}^{k} z_{2}^{l} \quad \text { for } \quad\left|z_{1}\right|=\left|z_{2}\right|=1,
$$

such that

$$
Q\left(z_{1}, z_{2}\right)=\left|P\left(z_{1}, z_{2}\right)\right|^{2}, \quad\left|z_{1}\right|=\left|z_{2}\right|=1
$$

Furthermore, we may not be able to write $Q\left(z_{1}, z_{2}\right)$ as a sum of squares of absolute values of some polynomials (see[1, 17]), which Calderon, Perpinsky, and Rudin prove.

To get a counterexample, we consider the Motzkin Polynomial

$$
Q\left(t_{1}, t_{2}\right)=t_{1}{ }^{4} t_{2}{ }^{2}+t_{1}{ }^{2} t_{2}{ }^{4}-3 t_{1}{ }^{2} t_{2}{ }^{2}+1 .
$$

By the arithmetic-geometric mean inequality, we see that,

$$
t_{1}^{4} t_{2}^{2}+t_{1}^{2} t_{2}^{4}+1 \geq 3 \sqrt[3]{{t_{1}}^{6} t_{2}{ }^{6}}=3 t_{1}{ }^{2} t_{2}{ }^{2}
$$

which implies that $Q\left(t_{1}, t_{2}\right)$ is nonnegative for all $t_{1}, t_{2} \in \mathbb{R}$. But this polynomial can not be represented as a sum of squares of polynomials. Indeed, if $Q\left(t_{1}, t_{2}\right)$ is a sum of squares of polynomials, that is, $Q\left(t_{1}, t_{2}\right)=\sum_{k} Q_{k}^{2}\left(t_{1}, t_{2}\right)$. Then each $Q_{i}\left(t_{1}, t_{2}\right)$ must have the form $a_{k} t_{1}^{2}+b_{k} t_{1}^{2} t_{2}+c_{k} t_{1}+d_{k} t_{1} t_{2}+$ $e_{k} t_{1} t_{2}^{2}+f_{k} t_{2}+g_{k} t_{2}^{2}+h_{k}$ i.e

$$
\begin{gathered}
Q\left(t_{1}, t_{2}\right)=\sum_{k}\left(a_{k} t_{1}^{2}+b_{k} t_{1}^{2} t_{2}+c_{k} t_{1}+d_{k} t_{1} t_{2}+e_{k} t_{1} t_{2}^{2}\right. \\
\left.+f_{k} t_{2}+g_{k} t_{2}^{2}+h_{k}\right)^{2} .
\end{gathered}
$$

By comparing the both sides of (56), we can see that Motzkin polynomial does not contain most of the terms which means some of these coefficients equal zero. The coefficient of $t_{1}^{4}$ in the right hand side of (56) must be zero, and therefore $\sum_{k} a_{k}^{2}=$ 0 and so $a_{k}=0$. Similarly we can obtain $g_{k}=c_{k}=f_{k}=0$. We have reduced $(56)$ to the form

$$
Q\left(t_{1}, t_{2}\right)=\sum_{k}\left(b_{k} t_{1}^{2} t_{2}+e_{k} t_{1} t_{2}^{2}+d_{k} t_{1} t_{2}+h_{k}\right)^{2} .
$$

The coefficient of $t_{1}^{2} t_{2}^{2}$ must be -3 i.e $\sum_{k} d_{k}^{2}=-3$ which is a contradiction. Therefore, the Motzkin polynomial is not any sum of squares of polynomials.

Theorem 10. Let $c_{k l},(k, l) \in\{0,1, \ldots, n\} \times\{0,1, \ldots, m\}$, then there exists a stable (no roots in $\bar{D}^{2}$ ) polynomial $P\left(z_{1}, z_{2}\right)=\sum_{l=0}^{m} \sum_{k=0}^{n} p_{k l} z_{1}^{k} z_{2}^{l}$ with $P_{00}>0$ such that the spectral density function of $P$ which is $f\left(z_{1}, z_{2}\right):=$ $\left(P\left(z_{1}, z_{2}\right) \overline{P\left(1 / \overline{z_{1}}, 1 / \overline{z_{2}}\right)}\right)^{-1}$ has Fourier coefficients $\hat{f}(k, l)=$ $c_{k l} \in\{1, \ldots, n\} \times\{-m, \ldots,-1\}$ if and only if there exists a complex number $c_{k l}(k, l) \in\{1, \ldots, n\} \times\{-m, \ldots,-1\}$ so that $(n+1)(m+1) \times(n+1)(m+1)$ doubly index Toeplitz matrix

$$
\Gamma=\left[\begin{array}{ccc}
C_{0} & \ldots & C_{n} \\
\vdots & \ddots & \vdots \\
C_{n} & \ldots & C_{0}
\end{array}\right]
$$

where

$$
C_{j}=\left[\begin{array}{ccc}
c_{j 0} & \ldots & c_{j,-m} \\
\vdots & \ddots & \vdots \\
c_{j m} & \ldots & c_{j 0}
\end{array}\right], \quad j=-n, \ldots, n
$$

and $c_{-k,-l}=\bar{c}_{k, l}$, has the following two properties:

\section{1. $\Gamma$ is positive definite.}

2. the $(n+1) m \times(m+1) n$ submatrix of $\Gamma$ obtained by removing scalar rows $1+j(m+1), j=0, \ldots, n$, and scalar columns $1,2, \ldots, m+1$, has rank $n m$.

In this case, one can find the column vector

$\left[p_{00}^{2} p_{00} p_{01} \ldots p_{00} p_{0 m} p_{00} p_{10} \ldots p_{00} p_{1 m} p_{00} p_{20} \ldots \ldots p_{00} p_{n m}\right]^{T}$,

as the first column of the inverse of $\Gamma$.

Let $c_{k}, k=0, \pm 1, \pm 2, \ldots, \pm n$ be a complex number,we can see that a stable polynomial, which has a degree $n$ and its spectral density function $f$, has Fourier coefficients $\hat{f}(k)=c_{k}, k=-n, \ldots, n$ if and only if the Toeplitz matrix $C:=\left(c_{i-j}\right)_{i, j=0}^{n}$ is positive definite (see [10]).

Theorem 11. Let

$$
Q\left(z_{1}, z_{2}\right)=\sum_{l=-m}^{m} \sum_{k=-n}^{n} q_{k l} z_{1}^{k} z_{2}^{l},
$$

such that

$$
Q\left(z_{1}, z_{2}\right)>0, \quad \text { for } \quad\left|z_{1}\right|=\left|z_{2}\right|=1 .
$$

Then there exists a polynomial

$$
P\left(z_{1}, z_{2}\right)=\sum_{l=0}^{m} \sum_{k=0}^{n} p_{k l} z_{1}^{k} z_{2}^{l},
$$

which is stable (i.e $P\left(z_{1}, z_{2}\right) \neq 0$, for $\bar{D}=\left\{z_{1}, z_{2} \in\right.$ $\left.\left.\mathbb{C} ;\left|z_{1}\right| \leq 1,\left|z_{2}\right| \leq 1\right\}\right)$ and $Q\left(z_{1}, z_{2}\right)=\left|P\left(z_{1}, z_{2}\right)\right|^{2}$ if and only if the matrix $\Gamma$ as in Theorem (10), the doubly index Toeplitz matrix obtained from the Fourier coefficients $c_{k}=$ $1 / Q(k, l)$ of the reciprocal of $Q$, satisfies condition 2 of Theorem (10), in this case the polynomial $P$ is unique up to multiplication with a complex number of absolute value equal 1 .

The Fejer-Riesz Theorem can be extended to several variables. Dritschel in [6] has proved that all strictly positive Laurent polynomials on the multi-torus can be factorized into a sum of squares of absolute values of polynomials.

Notation: Let $\mathbf{k}=\left(k_{1}, \ldots, k_{n}\right), e^{i \theta}=\left(e^{i \theta_{1}}, \ldots, e^{i \theta_{n}}\right)$, and $\mathbf{z}^{\mathbf{k}}=\left(z_{1}^{k_{1}}, \ldots, z_{n}^{k_{n}}\right)$. 
Theorem 12. Let $Q(z)=\sum_{k=-n}^{n} q_{k} \mathbf{z}^{\mathbf{k}}$ be a multivariate Laurent polynomial, such that $Q(z)>0$ on the multivariate torus $\left|z_{1}\right|=\left|z_{2}\right|=\ldots=\left|z_{n}\right|=1$, then there exists a polynomial $P(z)$ such that $Q(z)=P(z)^{*} P(z)$.

Dritschel gave proof based on the doubly infinite Toeplitz matrix. Geronimo and Lai provided another proof based on the univariate matrix Fejer-Riesz Theorem (see $[6,11])$.

\section{CONCLUSION}

This survey paper discussed the well-known Fejer-Riesz Theorem in different versions, such as the univariate and multivariate cases. We outlined the main ideas used in the proofs. In addition, we explained the critical conditions under which the factorization in Fejer-Riesz Theorem holds for the multivariate case.

\section{REFERENCES}

[1] A. Calderon and R. Pepinsky, On the phases of fourier coefficients for positive real periodic functions, Computing Methods and the Phase Problem in X-Ray Crystal Analysis (R. Pepinsky, ed.) (1950), 339-346.

[2] C.K. Chui, An Introduction to Wavelets, Academic Press, Boston (1992).

[3] C.K. Chui, Wavelets: A Mathematical Tool for Signal Analysis, SIAM, Philadelphia (1997).

[4] C. K. Chui, W. He and J. St ockler, Compactly supported tight and sibling frames with maximum vanishing moments, Appl. Comput. Harmon. Anal. 13 (2002), $224-262$

[5] I. Daubechies, Ten Lectures on Wavelets, CBMS-NSF Regional Conf. Series in Appl. Math., 61, Society for Industrial and Applied Mathematics, Philadelphia (1992).

[6] M.A. Dritschel, On factorization of trigonometric polynomials Integral Equations Operator Theory, 49 (2004), 11 - 42.

[7] M.A. Dritschel, J. Rovnyak, The operator Fejer- Riesz theorem, A Glimpse at Hilbert Space Operators: Paul R. Halmos in Memorium, Oper. Theory Adv. Appl., vol. 207, , Birkhauser Verlag, Basel (2010), 223 - 254.

[8] B. Dumitrescu, Positive Trigonometric Polynomials and Signal Procesing Applications, Dordrecht: Springer, 2007.

[9] L. Fejer, Uber trigonometrische Polynome, J. Reine Angew. Math 146 (1916), $53-82$.

[10] J.S. Geronimo, H.J. Woerdeman Positive extensions, Fejer- Riesz factorization and autoregressive filters in two variables Ann. of Math.(2),160 (3)(2004),839 - 906.

[11] J. S. Geronimo and Ming-Jun Lai, Factorization of multivariate positive Laurent polynomials, Journal of Approximation Theory 139 (2006) 327345 .

[12] D. Hardin, T. Hogen, Q. Sun, The matrix-valued Riesz Lemma and local orthonormal bases in shift-invariant spaces, Adv. Comput. Math., 20 (2004), pp. 367-384.

[13] F. Riesz, Uber ein Problem des Herr Caratheodory J. Reine Angew. Math., 146 (1915), 83 - 87.

[14] M. Rosenblum, Vectorial Toeplitz operators and Fejer- Riesz theorem,J. Math. Anal. Appl., 23 (1968), 139 - 147.

[15] M. Rosenblum, J. Rovnyak, Hardy Classes and Operator Theory, Oxford University Press, New York, 1985.

[16] M. Rosenblum, J. Rovnyak, The factorization problem for non-negative operator valued functions Bull. Am. Math. Soc., 77 (1971), 287 - 318.
[17] W. Rudin, The extension problem for positive-definite functions, Illinois J. Math. 7 (1963), 532 - 539.

\section{AUTHORS}

First Author - Abdulmtalb Hussen, Ph.D in Applied Mathematics, University of Missouri -St.Louis, United States: humu77@gmail.com.

Second Author - Abdelbaset Zeyani, Ph.D in Applied Mathematics , University of Missouri - St.Louis, United States: Alzayani1978@gmail.com. 